\title{
Standardized experimental estimation of the maximum unnoticeable environmental displacement during eye blinks for redirect walking in virtual reality.
}

\author{
Adrian Borrego \\ Neurorehabilitation and \\ Brain Research Group, i3b \\ Universitat Politècnica de \\ València \\ Valencia, Spain \\ adborgon@upv.es
}

\author{
Roberto Llorens \\ Neurorehabilitation and Brain \\ Research Group, i3ba \\ Universitat Politècnica de \\ València \\ Valencia, Spain \\ NEURORHB \\ Servicio de \\ Neurorrehabilitación de \\ Hospitales Vithas \\ Valencia, Spain \\ rllorens@i3b.upv.es
}

\begin{abstract}
Redirect walking is a technique that aims to manipulate the walking trajectories in immersive virtual reality settings by inducing unnoticeable displacements of the virtual environment. Taking into advantage the change blindness phenomenon, visual occlusion during eye blinks has been recently proposed to perform those displacements. This study determined the maximum unnoticeable displacement that can be performed in practical scenario, which proved to be near $0.8^{\circ}$ of occlusion and disocclusion in both horizontal and vertical axes.
\end{abstract}

Keywords - Redirect walking, virtual reality, change blindness.

\section{INTRODUCTION}

Implementation of real walking in virtual environments has always been a technological challenge for Virtual Reality (VR)-technology. Different alternatives have been proposed to facilitate more natural locomotion through the years, such as walking-in-place [1]. However, navigation in VR is still commonly enabled by more abstract metaphors using external keyboards or controllers, such as gamepads or joysticks. Although these user interfaces are worldwide known and used, and, over all, easier to implement, natural locomotion may be a requirement for rehabilitation application. This locomotion allows to maximize ecological validity of the training environment, and, ultimately, the transference of improvements from the virtual to the real world.

The last-generation of VR head mounted displays enable certain degree of free navigation as they include built-in tracking mechanisms that allows for estimating the position and orientation of the head in real time in a up to 5 meters area [2]. However, multi-camera solutions [3] are needed for wider spaces. Interestingly, room-size walkable VR solutions enables implementation of redirect walking (RDW) in VR [4]. RDW recreates the sensation of volitional real walking in any direction and distance of a virtual environment by rotating and translating the environment during walking. If changes of the virtual environment are sufficiently small and slow, they remain unnoticed to the users, which tricks their perception and, consequently, make them correct their trajectory to adapt

Ministerio de Economía y Competitividad of Spain (Project RTC-20176051-7, and Grant BES-2014-068218), Fundació la Marató de la TV3 (Grant 201701-10), and grant "Ayuda a Primeros Proyectos de Investigación (PAID06-18), Vicerrectorado de Investigación, Innovación y Transferencia de la Universitat Politècnica de València, València, Spain”.

\author{
Diego Gutierrez \\ Graphics and Imaging Lab \\ Universidad de Zaragoza \\ Zaragoza, Spain \\ diegog@unizar.es
}

\author{
Belen Masia \\ Graphics and Imaging Lab \\ Universidad de Zaragoza \\ Zaragoza, Spain \\ bmasia@unizar.es
}

to the updated environmental conditions. For instance, it is possible to make users believe that they are walking straight in an infinite path, while they are actually walking in circles [5]. Therefore, importance of RDW is that it allows recreating walkable virtual environments that are larger than the real environment itself.

The use of this technique has been reported by a number of interesting studies that have proved that RDW can be equivalent to real walking in terms of proprioceptive and subjective feedback [6]. Traditionally, manipulation of the virtual environment is smoothly and sequentially performed while users walk. Recent articles, however, have proposed that manipulation of the environment can also be performed during eye saccades [7] and blinks [8] increasing the range of applicable translations by approximately $10 \%$ Efficacy of using natural eye occlusion relies on the phenomena known as change blindness, which describes the inability to notice small changes that occur during visual disruption [9]. In spite of the potential of using visual occlusion to implement RDW techniques, it could be extremely difficult to put it into practice. Only one study has attempted to determine the maximum translation that could be actually done during an eye blink in VR [8]. However, the results of the study were only valid for the virtual environment used in the study and the results cannot be extrapolated. In addition to the lack of parametrization, the presence of different objects and textures could also produce motion parallax effects that could affect the results and were not controlled.

The objective of this study was to determine the maximum translation that can be performed in any virtual environment without being noticed during an eye blink, which would facilitate implementation of RDW in VR.

A. Participants

\section{METHODS}

A total of 45 healthy subjects participated in the study (27.9 \pm 7.1 years old, 30 men and 15 women). All of the participants provided written consent to participate in the study. 


\section{B. Instrumentation}

An HTC Vive Pro system (HTC Corporation, Taoyuan City, Taiwan) was used to display the VE. An eye-tracking system, the Pupil (Pupil Labs, Berlin, Germany) was embedded into the HMD using the HTC Vive Binocular Addon of the same manufacturer. The Pupil includes two $120 \mathrm{~Hz}-$ cameras, each one illuminated by 10 IR LEDs.

The virtual environment consisting of a cloud of random black dots over a white background and a white panel with a black cross on its center, which was initially located between the user and the background (Fig. 1).

Fig. 1. a) Virtual environment displayed during the experiment; b) and c) are the different possible displacements of the virtual environement. Avatar is a representation for the virtual camera position.

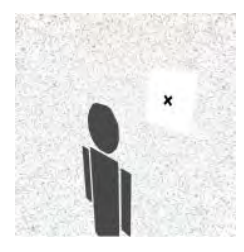

a)

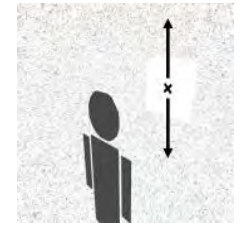

b)

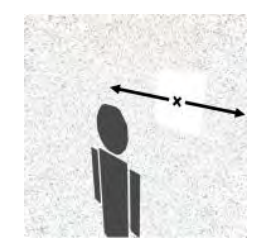

c)
The VE was implemented and rendered using Unity3D v2018.1 (Unity Technologies ApS, San Francisco, CA) and blinks were detected using the Pupil Capture Software v1.9-7.

\section{Procedure}

Initially, participants were asked to comfortably sit in a chair, were informed about the objective of the experiment, were equipped with the head mounted display, and were asked to remain as still as possible. Two conditions were designed to detect the maximum noticeable horizontal or vertical displacement. For both conditions, participants were asked to look at the black cross of the panel and follow the instructions given during the experiment. A dedicated program detected the beginning of the eye blinks, with a maximum delay of $93.8 \mathrm{~ms}$, and translated the whole virtual environment horizontally or vertically, depending on the condition, in one direction or the other, or remain the same. Three seconds after a blink, a message appeared asking participants whether or not they had detected any change. An experimenter introduced each answer into a Quest staircase algorithm [10] implemented in the program, which estimated the translation to be made during the next eye blink. The experiment finished when the difference between the last two environmental translations created an angle occlusion smaller than $0.1^{\circ}$.

The maximum background angle that was successfully occluded or disoccluded during the experiment without being noticed was estimated for each condition (Fig. 2).

Fig. 2. Angle disoccluded after a left displacement.

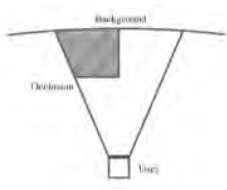

a)

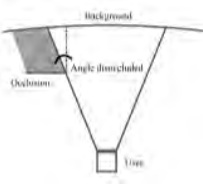

b)

\section{RESULTS}

The mean maximum angle that could be performed without being noticed was $0.82 \pm 0.31^{\circ}$ in the horizontal axis and 0.80 $\pm 0.29^{\circ}$ in the vertical axis.

\section{DISCUSSION}

This study determines the maximum translation that can be made in a virtual environment without being perceived in both the horizontal and vertical axis in angular measures that allow extrapolation to any practical scenario. By using a simple environment were only motion parallax effect is present, experimental results do not depend on the depth or size of the occlusion. Human perception seems to present limitations to detect occlusions and disocclusions near $0.8^{\circ}$ in both horizontal and vertical axis. Considering a practical scenario that consists of a wall located 4 meters away from the user and an occluding object situated in the centre point between the user and the wall, the whole environment could be translated $3.85 \mathrm{~cm}$ horizontally and $3.72 \mathrm{~cm}$ vertically without being perceived.

In conclusion, the noticeable thresholds estimated in this study could be used in future implementations of RDW techniques in VR to maximize the environmental translations that can be applied without being noticed during eye blinks.

\section{REFERENCES}

[1] F. Steinicke, G. Bruder, K. Hinrichs, and A. Steed, "Presence-enhancing real walking user interface for first-person video games," in Proceedings of the 2009 ACM SIGGRAPH Symposium on Video Games - Sandbox '09, 2009.

[2] A. Borrego, J. Latorre, M. Alcañiz, and R. Llorens, "Comparison of Oculus Rift and HTC Vive: Feasibility for Virtual Reality-Based Exploration, Navigation, Exergaming, and Rehabilitation," Games Health J., 2018.

[3] J. C. P. Chan, H. Leung, J. K. T. Tang, and T. Komura, "A virtual reality dance training system using motion capture technology," IEEE Trans. Learn. Technol., 2011.

[4] S. Razzaque, Z. Kohn, and M. C. Whitton, "Redirected Walking," EUROGRAPHICS, 2001.

[5] F. Steinicke, G. Bruder, T. Ropinski, and K. H. Hinrichs, "Moving Towards Generally Applicable Redirected Walking," Proc. IEEE VRIC 2008 10th Int. Conf. Virtual Real., 2008.

[6] F. Steinicke, G. Bruder, J. Jerald, H. Frenz, and M. Lappe, "Estimation of detection thresholds for redirected walking techniques," IEEE Trans. Vis. Comput. Graph., 2010.

[7] Q. Sun et al., "Towards Virtual Reality Infinite Walking: Dynamic Saccadic Redirection,” ACM Trans. Graph., 2018.

[8] E. Langbehn, F. Steinicke, M. Lappe, G. F. Welch, and G. Bruder, "In the blink of an eye: leveraging blink-induced suppression for imperceptible position and orientation redirection in virtual reality," ACM Trans. Graph., vol. 37, no. 4, pp. 1-11, Jul. 2018.

[9] D. J. Simons and D. T. Levin, "Failure to detect changes to people during a real-world interaction,” Psychon. Bull. Rev., 1998.

[10] A. B. Watson and D. G. Pelli, "Quest: A Bayesian adaptive psychometric method,” Percept. Psychophys., 1983. 